\title{
Optical Power Budget Enhancement in 50-90 Gb/s IM-DD PONs with NOMA-CAP and SOA-based Amplification
}

\author{
Samael Sarmiento, Student Member, IEEE, José Manuel Delgado Mendinueta, Member, IEEE, \\ José Antonio Altabás, Salvatore Spadaro, Senior Member, IEEE, Satoshi Shinada, Member, IEEE, \\ Hideaki Furukawa, Member, IEEE, Juan José Vegas Olmos, Senior Member, IEEE, \\ José Antonio Lázaro, Member, IEEE, and Naoya Wada, Member, IEEE
}

\begin{abstract}
We report experimental results of a semiconductor optical amplifier (SOA) for optical amplification in a 50-90 Gb/s IM-DD optical access system using non-orthogonal multiple access multiplexing combined with multi-band carrierless amplitude phase modulation format (NOMACAP). Specifically, the SOA has been investigated as both booster and preamplifier for downstream applications in PONs. The measured optical power budgets resulting from performing booster amplification and preamplification simultaneously, assuming a BER threshold at $2.2 \times 10^{-3}$, are 28.7(23.7) $\mathrm{dB}$ and 23.6(18.9) $\mathrm{dB}$ at $\mathbf{5 0}(90) \mathrm{Gb} / \mathrm{s}$ for the strong and weak NOMA levels, respectively, compared to the budgets for the strong and weak NOMA levels of 12.4(8.7) $\mathrm{dB}$ and $9.4(5.2) \mathrm{dB}$ when no optical amplification is used.
\end{abstract}

Index Terms-Multi-band carrierless amplitude phase modulation, non-orthogonal multiple access, semiconductor optical amplifier, passive optical networks, optical interconnects.

\section{INTRODUCTION}

The information and communications technology (ICT) ecosystem has been changing rapidly over the last years. New multimedia and cloud services, along with the deployment of the internet of things (IoT) and the convergence between optical and wireless communications, demand changes to the access networks to enable a scalable growth in traffic volume, while supporting a high level of dynamic connectivity, full network flexibility, reduced end-to-end delay and improved energy-efficiency. Traditionally, these systems rely on non-return to zero (NRZ) and lately 4-level pulse-amplitude modulation (PAM-4) to encode the binary streams in the link; however, scaling beyond $25 \mathrm{~Gb} / \mathrm{s}$ in access systems where cost is a key parameter indicator is challenging. Orthogonal frequency division multiplexing (OFDM) has been extensively explored as a potential candidate for future next generation passive optical networks (PONs) due to its numerous benefits such as high spectral efficiency, high resilience to chromatic dispersion (CD) and efficient/flexible resource management. Nevertheless, the main drawback of OFDM is its high computational complexity [1]. To overcome this problem, multi-band carrierless amplitude phase (multi-CAP) modulation format has been investigated [2].

Manuscript received MM DD, AA; revised MM DD, AA; accepted MM DD, AA. This work was supported in part by ALLIANCE (TEC2017-90034C2-2-R) project co-funded by FEDER, the European Union's Horizon 2020 research and innovation programme under grant agreement no 780997 (plaCMOS), as well as MINECO FPI-BES-2015-074302.

Samael Sarmiento, Salvatore Spadaro and José Antonio Lázaro are with the School of Telecommunications Engineering, Polytechnic University of Catalonia, Barcelona 08034, Spain (e-mail: samael.sarmiento@tsc.upc.edu; spadaro@tsc.upc.edu; jose.lazaro@tsc.upc.edu).

José Manuel Delgado Mendinueta, Satoshi Shinada, Hideaki Furukawa and Naoya Wada are with the Photonic Network System Laboratory, National
Non-orthogonal multiple access (NOMA) has recently been proposed as an alternative solution to increase the spectral efficiency, enabled by multiplexing different users in the power domain. Successive interference cancellation (SIC) must be conducted at the receiver for the multi-user signal separation [3], which increases the receiver complexity for a high number of users. To mitigate this issue, redundancy can be added to the users signals through spectrum spreading or coding techniques since it facilitates the user's signal separation, but decreasing the spectral efficiency [4]. Alternatively, SIC can be implemented with moderate complexity, without the need for spreading/coding redundancy, by limiting the number of powermultiplexed users to two and the constellation alphabet to four symbols, as demonstrated in [5]. Moreover, SIC with moderate complexity can be implemented using available technology with a tolerable increase of the energy consumption [6].

NOMA and multi-CAP working in tandem, named NOMA-CAP, have been previously reported in [7] and demonstrates flexible multiuser provisioning with a total aggregated capacity of $30 \mathrm{~Gb} / \mathrm{s}$ and an optical link span limited to $10 \mathrm{~km}$ due to the low available optical power budget. Moreover, NOMA-CAP has been recently reported in [8], demonstrating its feasibility for short range $(<2 \mathrm{~km})$ optical transmission applications such as $5 \mathrm{G}$ mobile fronthaul and data center interconnects, where limited available optical power budgets are the norm. Hence, to increase both the data rate and the optical reach by increasing the optical power budget, NOMA-CAP must be combined with optical amplification, including semiconductor optical amplifiers (SOAs) or erbium doped fiber amplifiers (EDFAs). Recently, SOAs have attracted more attention due to the large optical bandwidth for booster amplification and pre-amplification applications in 25G EPON and beyond [9, 10], besides the small size and the ability to be integrated with other optical devices. Nevertheless, SOAs may have an impact on the data pattern quality due to its fast-dynamic gain, i.e., the patterning effect, and nonlinearities [10]. Alternatively, avalanche photodiodes (APDs) can also be used to increase the optical power budget of the link. However, bandwidth of low-cost APDs is still limited to $10 \mathrm{GHz} .18 \mathrm{GHz}$ bandwidth APDs are becoming available in the market but their high cost makes them prohibitive [11]. NOMACAP and SOA-based amplification have been investigated in [12]

Institute of Information and Communication Technology (NICT), 4-2-1 NukuiKitamachi, Koganei, Tokyo 184-8795, Japan.(e-mail: mendi@nict.go.jp, sshinada@nict.go.jp, furukawa@nict.go.jp,wada@nict.go.jp).

José Antonio Altabás is with Bifrost Communications, Scion DTU, Akademivej Bygnig 381, $2800 \mathrm{Kgs}$ Lyngby, Denmark, and the Aragon Institute of Engineering Research, University of Zaragoza, Zaragoza 50018, Spain (email: jaltabas@unizar.es).

Juan José Vegas Olmos is with Mellanox Technologies, Yokneam, 20692, Israel (e-mail: juanj@mellanox.com). 


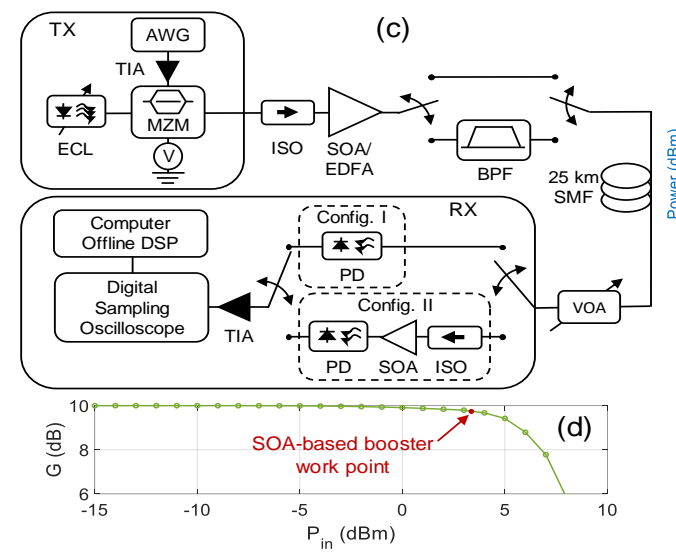

(e)



Fig. 1. NOMA-CAP DSP block diagram for (a) the digital transmitter (DTX) and (b) digital receiver (DRX). (c) Experimental PON setup. (Acronyms) ECL: external cavity laser, V: voltage source, AWG: arbitrary waveform generator, ISO: isolator, EDFA: erbium doped fiber amplifier, BPF: band pass filter, VOA: variable optical attenuator, PD: photodiode, TIA: transimpedance amplifier. (d) Optical gain (G) vs. input power $\left(\mathrm{P}_{\text {in }}\right)$ characterization of used SOA. (e) Received NOMA-CAP spectra for 9 CAP bands, providing an aggregated bit rate of $90 \mathrm{~Gb} / \mathrm{s}$, as well as the IM-DD channel frequency response for $25 \mathrm{~km}$ SMF. (f) Received constellations at the considered FEC limit for $25 \mathrm{~km} \mathrm{SMF} \mathrm{configuration} \mathrm{of} \mathrm{the} \mathrm{strong} \mathrm{and} \mathrm{weak} \mathrm{NOMA} \mathrm{levels} \mathrm{for} 9$ CAP bands. Red circles are the constellation centroids.

showing promising results with a total aggregate capacity of $50 \mathrm{~Gb} / \mathrm{s}$ in back-to-back configuration. In this paper, NOMA-CAP modulation, providing a total aggregated capacity from $50 \mathrm{~Gb} / \mathrm{s}$ to $90 \mathrm{~Gb} / \mathrm{s}$, has been assessed for downstream applications in a PON scenario with an optical span of $25 \mathrm{~km}$ when a SOA is used as both booster and preamplifier. Specifically, using intensity modulation with direct detection (IM-DD) as the simplest and most cost-effective solution, we characterize the SOA with NOMA-CAP signals with data rates ranging from 50 to $90 \mathrm{~Gb} / \mathrm{s}$ in terms of the sensitivity of a PIN-based receiver. For comparison purposes, an EDFA for booster amplification applications has also been investigated. Experimental results demonstrate that, assuming a 7\% overhead $(\mathrm{OH})$ for forward error corrections (FEC) with the BER threshold at $2.2 \times 10^{-3}$, the optical power budgets are higher than 23.6(18.9) $\mathrm{dB}$ at 50(90) $\mathrm{Gb} / \mathrm{s}$ when both booster and pre-amplifier are used and budgets lower than $12.4(8.7) \mathrm{dB}$ when no optical amplification is performed. Note that higher power budgets could be achieved using more powerful FEC techniques, such as low-density parity-check (LDPC) codes used in $25 \mathrm{G}$ EPON that considers a BER threshold of $1 \times 10^{-2}$, but at the cost of increasing the de/coder complexity. In this case, for $50 \mathrm{~Gb} / \mathrm{s}$ the power budget for the strong and weak NOMA levels are close to $29 \mathrm{~dB}$, corresponding to the PR-30 requirement in $10 \mathrm{G}$ EPON.

The remainder of this paper is organized as follows. Section II describes the NOMA-CAP digital signal processing (DSP) for signal generation and detection when two NOMA levels and different number of CAP bands of $2.5 \mathrm{GHz}$ are considered, and the setup of the implemented optical system model. Section III shows the experimental characterization for different bit rates. Finally, Section IV concludes the paper and draws the main conclusions.

\section{Experimental setup description}

\section{A. NOMA-CAP signal generation and detection}

The transmitter DSP architecture for NOMA-CAP transmission with two NOMA levels per band is shown in Fig. 1(a). First, the data of both NOMA levels were distributed among all the multi-CAP bands and mapped to quaternary quadrature amplitude modulation (4-QAM) symbols. The two NOMA levels, named "weak NOMA" and "strong NOMA", were power-weighted according to the power ratio $r_{p}$ (defined as the relation between the two NOMA levels, $r_{p}=$ $\left.20 \log _{10}\left(w_{\text {strong }} / w_{\text {weak }}\right)\right)$, and added for each multi-CAP band. The multi-CAP signals were upsampled and filtered with a pair of band- specific multi-CAP orthogonal filters and finally the multi-CAP bands were aggregated to form the full data transmitted signal [7]. A squareroot raised cosine (SRRC) filter with a roll off factor equal to 0.2 was used as pulse shaper. In this work, two NOMA levels with varying numbers of $2.5 \mathrm{GHz}$ multi-CAP bands, $N_{\text {bands }}=\{5,7,9\}$, corresponding to bit rates $R_{b}=\{50,70,90\} \mathrm{Gb} / \mathrm{s}$, were assessed. Both the SRRC filter roll off factor and the multi-CAP band bandwidth were empirically chosen to reduce the effect of the power fading dips of IMDD transmission systems due to CD. Finally, it was established a $r_{p}=$ $7 \mathrm{~dB}$ as a good trade-off that optimizes the reception of both NOMA levels for all the considered total aggregated bit rates [12].

The receiver DSP block diagram is shown in Fig. 1(b). The first step was the low-pass filtering of the digitized signal with a finite impulse response (FIR) filter to reduce noise, and then each multi-CAP band was extracted using the inverse pair of orthogonal filters used at the transmitter for the band of interest [7]. For the weak NOMA level, it is necessary to implement SIC as shown in Fig. 1(b), consisting of a decision feedback equalizer (DFE) with 30 forward and 20 backward taps, calculation of the symbol centroid of the strong NOMA level and subtraction from the equalized signal. After SIC, the DFE was applied again to the resulting signal and finally the signal was de-mapped. For the strong NOMA level, only the DFE and the de-mapping were performed. In both cases, a training sequence was used to determine the DFE filter coefficients. Moreover, since the channel delay spread was on the order of the symbol duration, any DFE backpropagation error was observed. In case of the DFE backpropagation error could degrade the system performances, Tomlinson-Harashima precoding (THP), which places the feedback equalizer at the transmitter, can be adopted, reducing receiver complexity as well [13].

\section{B. PON experimental setup}

The experimental setup is depicted in Fig. 1(c). The optical transmitter (TX) was based on an external cavity laser (ECL) tuned at $1548 \mathrm{~nm}$ and modulated with a Mach-Zehnder modulator (MZM) biased at its quadrature point. Although an ECL was used, a distributed feedback (DFB) laser can also be considered to reduce implementation costs since the increased phase noise of the DFB compared to the ECL does not affect the system performance. The MZM was driven with an arbitrary waveform generator (AWG) with $20 \mathrm{GHz} 3 \mathrm{~dB}$ electrical bandwidth to generate the NOMA-CAP modulation. The modulated optical signal was then amplified at the TX output by a SOA or an EDFA. The SOA bandwidth was $70.9 \mathrm{~nm}$, centered around $1497.7 \mathrm{~nm}$, 


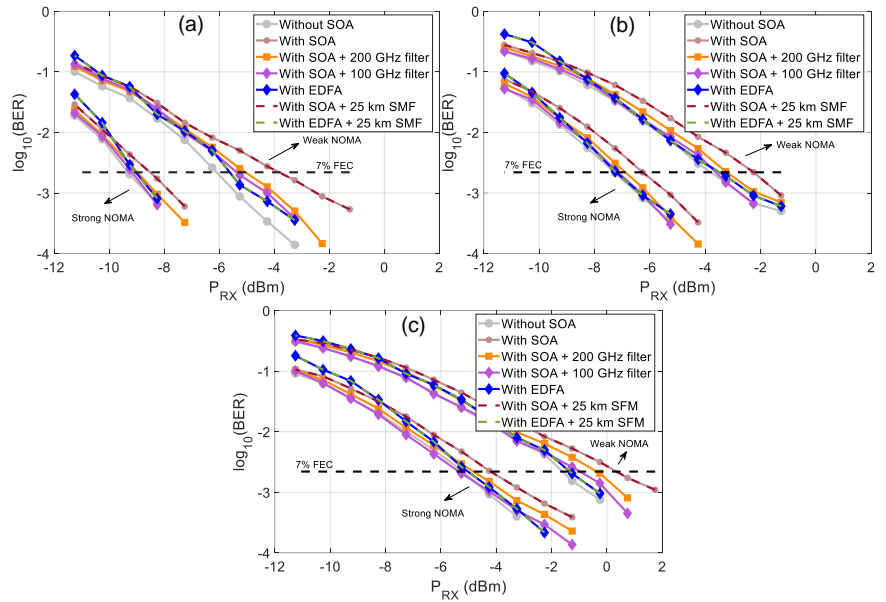

Fig. 2. BER vs. received power $\left(P_{R X}\right)$ for the strong and weak NOMA levels when the optical signal is amplified by SOA/EDFA and optionally filtered with $0 \mathrm{~km}$ and $25 \mathrm{~km}$ of SMF for: (a) $50 \mathrm{~Gb} / \mathrm{s}$, (b) $70 \mathrm{~Gb} / \mathrm{s}$ and (c) $90 \mathrm{~Gb} / \mathrm{s}$.

the saturation output power was $+16 \mathrm{dBm}$, the noise figure $(\mathrm{NF})$ was $8.4 \mathrm{~dB}$ and the measured gain was $10 \mathrm{~dB}$ for an input power of $0 \mathrm{~dB}$, as shown in Fig. 1(d). The EDFA bandwidth was $35 \mathrm{~nm}$ centered at $1548 \mathrm{~nm}$, the saturation output power was $+28 \mathrm{dBm}$, the NF was $4.5 \mathrm{~dB}$ and the gain was $18 \mathrm{~dB}$ at an input power of $0 \mathrm{dBm}$. The optical power at the transmitter output was $+3.4 \mathrm{dBm}$ for which the SOA is still operating in the linear regime, as shown in Fig. 1(d). The amplified signal was optionally filtered by a $100(200) \mathrm{GHz}$ band pass filter to investigate the effect of the amplified spontaneous emission (ASE) noise over the optical signal. The optical signal was then launched into a $25 \mathrm{~km}$ span of single-mode fiber (SMF). Two configurations were considered for the optical receiver (RX). The first one based on a PIN photodetector (PD) combined with an electrical amplifier (RX Config. I, refer to Fig. 1(c)), and the second one, a pre-amplified receiver with the same PD (RX Config. II, in Fig. 1(c)). A SOA with similar characteristics to the one used in the TX output was used in the preamplified receiver configuration. The detected signal was finally digitalized with a $100 \mathrm{GSa} / \mathrm{s}$ real time oscilloscope with $33 \mathrm{GHz}$ of electrical bandwidth and processed off-line.

\section{Experimental results and discussion}

Fig. 1(e) depicts the received NOMA-CAP spectra after $25 \mathrm{~km}$ SMF for $N_{\text {bands }}=9$, corresponding to $R_{b}=90 \mathrm{~Gb} / \mathrm{s}$. There, it can be observed that the required electrical bandwidth for $N_{\text {bands }}=9$ is $27 \mathrm{GHz}$, while for $N_{\text {bands }}=\{5,7\}$, corresponding to $R_{b}=\{50,70\} \mathrm{Gb} / \mathrm{s}$, is $15 \mathrm{GHz}$ and $21 \mathrm{GHz}$, respectively. Fig. 1(e) also depicts the frequency response of the $25 \mathrm{~km}$ SMF channel for the IM-DD system proposed in Fig. 1(c). In Fig. 1(e), it can be observed that the frequencies of the fading dips due to $\mathrm{CD}$ are between two different bands, overcoming therefore the bandwidth limitation of the

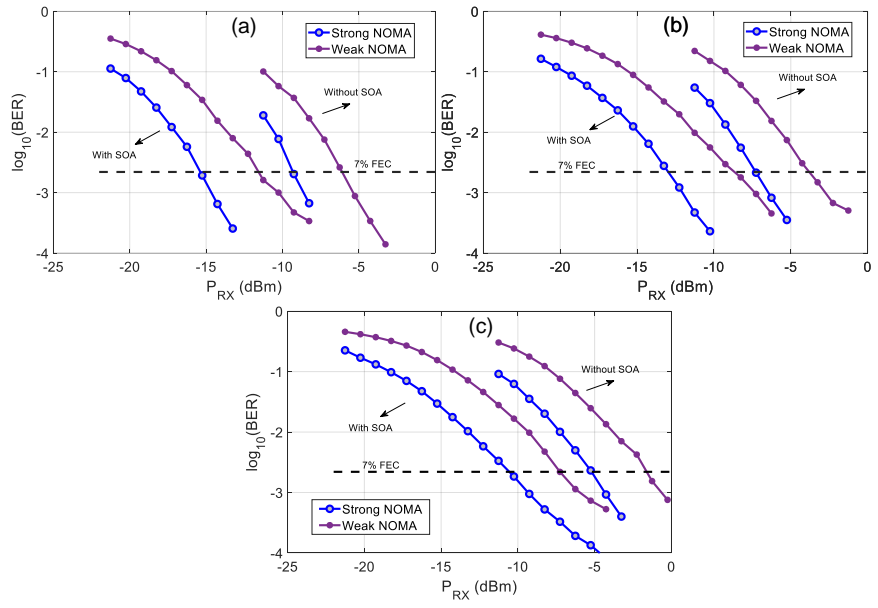

Fig. 3. BER vs. received power $\left(P_{R X}\right)$ comparison when the NOMA-CAP signal is pre-amplified by a SOA at the receiver and not with $25 \mathrm{~km}$ SMF for: (a) $50 \mathrm{~Gb} / \mathrm{s}$, (b) $70 \mathrm{~Gb} / \mathrm{s}$ and (c) $90 \mathrm{~Gb} / \mathrm{s}$.

transmission channel for IM-DD systems. Finally, Fig. 1(f) shows the received constellations on each band and each NOMA level at the considered FEC limit with $25 \mathrm{~km}$ SMF.

Fig. 2 shows the BER vs. $P_{R X}$ curves for the strong and weak NOMA levels for $R_{b}=\{50,70,90\} \mathrm{Gb} / \mathrm{s}$ and for the cases in which the NOMA-CAP signal has been optically boosted by the SOA/EDFA at the transmitter output and with no optical amplification. In these measurements, the optical receiver was only based on a PD (see Fig. 1(c), Config. I). To determine the effect of the SOA-induced ASE noise over the receiver sensitivities, Fig. 2 also shows the BER curves when the optical signal is filtered at the SOA output with an optical band pass filter with bandwidths of 100 or $200 \mathrm{GHz}$. When using the EDFA, no optical filtering is required since a high optical signal to noise ratio (OSNR) is obtained $(>40 \mathrm{~dB})$. Table I summarizes the receiver sensitivities depicted in Fig. 2 for $R_{b}=\{50,70,90\} \mathrm{Gb} / \mathrm{s}$. For both the strong and weak NOMA levels, the sensitivity penalty when the bit rate is increased from $50 \mathrm{~Gb} / \mathrm{s}$ to $70 \mathrm{~Gb} / \mathrm{s}$ is close to $2 \mathrm{~dB}$, while increasing the bit rate from $50 \mathrm{~Gb} / \mathrm{s}$ to $90 \mathrm{~Gb} / \mathrm{s}$ increments the penalty about $4 \mathrm{~dB}$. This penalty degradation is due to the electrical bandwidth limitation at the transmitter and receiver sides, see Fig. 1(e). From Table I, the averaged penalty $\bar{\Delta}$ (defined as the average receiver sensitivity differences among all bit rates) is of $0.8 \mathrm{~dB}$ and $1.8 \mathrm{~dB}$ for the strong and weak NOMA levels when the SOA is used without optical band pass filtering, respectively, while the penalties over the receiver sensitivity of the strong and the weak NOMA levels when the EDFA is used are just $0.1 \mathrm{~dB}$ and $0.4 \mathrm{~dB}$, respectively. For the SOA plus filtering case, $\bar{\Delta}$ for the strong and weak NOMA levels are now reduced to $0.1(0.3) \mathrm{dB}$ and $0.7(1.0) \mathrm{dB}$ when the $100(200) \mathrm{GHz}$ bandwidth band pass filter is used, respectively. Clearly, the penalty reduction is due to ASE noise reduction in the optical filter. Despite those small penalties, the SOA and EDFA provide output power gains of $10 \mathrm{~dB}$ and $18 \mathrm{~dB}$, respectively. Finally, from Fig. 2, no penalty was

TABLE I

SUMMARY OF RECEIVER SENSITIVITIES (SS) OF THE WEAK AND STRONG NOMA LEVELS FOR $R_{b}=\{50,70,90\}$ GB/S WHEN THE SIGNAL IS AMPLIFIED BY SOA/EDFA, OPTICALLY BAND PASS FILTERED AND THE RECEIVER IS BASED ON A PIN PD AS WELL AS THEIR POWER PENALTY $(\Delta)$ COMPARED TO THE CASE IN WHICH NO OPTICAL AMPLIFICATION IS USED. $\bar{\Delta}$ IS THE AVERAGED POWER PENALTY AMONG ALL CONSIDERED BIT RATES.

\begin{tabular}{|c|c|c|c|c|c|c|c|c|c|c|c|}
\hline \multirow{3}{*}{$R_{b}(G b / s)$} & \multirow{3}{*}{$\begin{array}{c}\text { NOMA level } \\
\text { Optical amplifier } \\
\text { BW filter }(\mathrm{GHz})\end{array}$} & \multicolumn{5}{|c|}{ Strong } & \multicolumn{5}{|c|}{ Weak } \\
\hline & & No & \multicolumn{3}{|c|}{ SOA } & \multirow{2}{*}{$\begin{array}{c}\text { EDFA } \\
\infty\end{array}$} & \multirow{2}{*}{$\begin{array}{c}\text { No } \\
\infty\end{array}$} & \multicolumn{3}{|c|}{ SOA } & \multirow{2}{*}{$\frac{\text { EDFA }}{\infty}$} \\
\hline & & $\infty$ & 100 & 200 & $\infty$ & & & 100 & 200 & $\infty$ & \\
\hline \multirow{2}{*}{50} & $\mathrm{~S}(\mathrm{dBm})$ & -9.0 & -8.9 & -8.8 & -8.5 & -8.9 & -6.0 & -5.2 & -5.1 & -4.0 & -5.6 \\
\hline & $\Delta(\mathrm{dB})$ & & 0.1 & 0.2 & 0.5 & 0.1 & & 0.8 & 0.9 & 2.0 & 0.4 \\
\hline \multirow[b]{2}{*}{70} & $\mathrm{~S}(\mathrm{dBm})$ & -7.3 & -7.2 & -7.1 & -6.3 & -7.2 & -3.8 & -3.6 & -3.3 & -2.3 & -3.5 \\
\hline & $\Delta(\mathrm{dB})$ & & 0.1 & 0.4 & 1.0 & 0.1 & & 0.7 & 0.5 & 1.5 & 0.3 \\
\hline \multirow[b]{2}{*}{90} & $\mathrm{~S}(\mathrm{dBm})$ & -5.3 & -5.1 & -4.9 & -4.3 & -5.1 & -1.8 & -1.2 & -0.3 & 0.2 & -1.3 \\
\hline & $\Delta(\mathrm{dB})$ & & 0.2 & 0.4 & 1.0 & 0.2 & & 0.6 & 1.5 & 2.0 & 0.5 \\
\hline \multicolumn{2}{|c|}{$\bar{\Delta}(\mathrm{dB})$} & & 0.1 & 0.3 & 0.8 & 0.1 & & 0.7 & 1.0 & 1.8 & 0.4 \\
\hline
\end{tabular}


TABLE II

SUMMARY OF AVAILABLE POWER BUDGET ( $P B$ ) OF THE WEAK AND STRONG NOMA LEVELS FOR $R_{b}=\{50,70,90\}$ GB/S WHEN NO OPTICAL AMPLIFICATION IS PERFORMED AND WHEN SOA-BASED AMPLIFICATION IS USED AT THE TRANSMITTER SIDE (BOOSTER), AT THE RECEIVER SIDE (PRE-AMPLIFIER), AND AT BOTH SIDES ASSUMING A 7\% FEC OH.

\begin{tabular}{|c|c|c|c|c|c|c|}
\hline \hline NOMA level & \multicolumn{2}{|c|}{ Strong $P B(\mathrm{~dB})$} & \multicolumn{3}{|c|}{ Weak $P B(\mathrm{~dB})$} \\
\hline$R_{b}(G b / s)$ & 50 & 70 & 90 & 50 & 70 & 90 \\
\hline Without optical amplification & 12.4 & 10.7 & 8.7 & 9.4 & 7.2 & 5.2 \\
\hline With booster & 22.4 & 20.2 & 18.2 & 17.9 & 16.2 & 13.7 \\
\hline With pre-amplifier & 18.7 & 16.7 & 14.2 & 15.1 & 12.7 & 10.4 \\
\hline With both booster and pre-amplifier & 28.7 & 26.2 & 23.7 & 23.6 & 21.7 & 18.9 \\
\hline
\end{tabular}

observed in the receiver sensitivity after $25 \mathrm{~km}$ transmission when the optical signal is amplified by the SOA or EDFA due to the high resilience of multi-CAP to CD [2], since the SRRC roll off factor and the multi-CAP band bandwidth can be chosen to overcome the penalty caused by the CD.

Fig. 3 shows the measured sensitivity enhancement in the receiver when the proposed PD-based receiver is upgraded with a SOA in a preamplifier configuration, as shown in Fig. 1(c) (Config. II), and no amplification is performed at the transmitter output for $R_{b}=$ $\{50,70,90\} \mathrm{Gb} / \mathrm{s}$ and a $25 \mathrm{~km}$ of SMF. Now, the BER is measured in terms of the input power to the SOA at the receiver. This leads to an OSNR variation which is different from the previously studied cases, resulting therefore in different BER curves. The new receiver sensitivities are, for the strong NOMA level, $-15.3 \mathrm{dBm},-13.3 \mathrm{dBm}$ and $-10.8 \mathrm{dBm}$ for $50 \mathrm{~Gb} / \mathrm{s}, 70 \mathrm{~Gb} / \mathrm{s}$ and $90 \mathrm{~Gb} / \mathrm{s}$, respectively, while, for the weak NOMA level are $-11.7 \mathrm{dBm},-9.3 \mathrm{dBm}$ and $-7.0 \mathrm{dBm}$ for $50 \mathrm{~Gb} / \mathrm{s}, 70 \mathrm{~Gb} / \mathrm{s}$ and $90 \mathrm{~Gb} / \mathrm{s}$, respectively. Therefore, for the strong and weak NOMA levels, the receiver sensitivity enhancements are close to $6.0 \mathrm{~dB}$ and $5.5 \mathrm{~dB}$, respectively. Higher enhancements cannot be achieved since the ASE noise of the SOA degrades the output OSNR. In these measurements, optical filtering to reduce the impact of the ASE noise has not been performed to reduce costs at the receiver. Therefore, future SOA-PD photonic integrated circuits (PICs) could be a very suitable solution.

Finally, given all the measured sensitives and considering that the transmitter output power is $+3.4 \mathrm{dBm}$, Table II shows the available optical power budgets $(P B)$ for the strong and weak NOMA levels when no optical amplification is used, and when SOA-based amplification is performed at the transmitter side (booster), at the receiver side (pre-amplifier) and at both sides for $R_{b}=\{50,70$, $90\} \mathrm{Gb} / \mathrm{s}$. If optical filtering is performed at the booster SOA, i.e., emulating a WDM-based PONs, the power budget of the strong NOMA level decreases in $5 \mathrm{~dB}$ (typical insertion loss of an arrayed waveguide grating (AWG)-based de/multiplexer) while for the weak NOMA level it is reduced by $4 \mathrm{~dB}$ due to the gain of an approximately $1 \mathrm{~dB}$ obtained when the ASE noise is filtered. From Table II, it can be observed a $P B$ reduction of $3.5 \mathrm{~dB}$ when the pre-amplification configuration is used regarding the booster configuration. This is due to the fact that in the booster configuration the OSNR remains always constant while in the pre-amplification configuration, the optical signal power is varied at the SOA input causing a degradation of the OSNR before the PD. An enhancement of $15.6 \mathrm{~dB}$ and $14.2 \mathrm{~dB}$ in the $P B$ can also be observed in Table II for the strong and weak NOMA levels, respectively, when both booster and pre-amplifier are used compared with no optical amplification case.

\section{Conclusion}

In this paper, 50-90 Gb/s NOMA-CAP with two power levels has been experimentally measured in an IM-DD system with a PIN-based receiver and $25 \mathrm{~km}$ of SMF for PON downstream applications. To increase the low available optical power budget of the system, SOAbased amplification at the transmitter and receiver sides has been assessed. Two main conclusions can be extracted from this experimental assessment. First, the SOA shows better performance in terms of power budget as a booster since the system enhancement is
$3.5 \mathrm{~dB}$ compared to the pre-amplifier case. Second, obtaining high power budgets requires both boosters and pre-amplifiers. In this case, SOA-based amplification for NOMA-CAP modulation provides optical power budgets of 28.7(23.7) dB and 23.6(18.9) dB at $50(90) \mathrm{Gb} / \mathrm{s}$ for the strong and weak NOMA levels, respectively, while the budgets for the strong and weak NOMA levels are 12.4(8.7) dB and 9.4(5.2) $\mathrm{dB}$ at 50(90) $\mathrm{Gb} / \mathrm{s}$ when no optical amplification is used, assuming a $7 \%$ FEC OH. Finally, we consider that NOMA-CAP with SOA-based amplification, e.g., offering a maximum optical splitting ratio of $64(32)$ at $50(90) \mathrm{Gb} / \mathrm{s}$ for an optical reach between $25(24.8) \mathrm{km}$ and $10.4(5.6) \mathrm{km}$, could be a good modulation format candidate for next generation PONs. Particularly, we believe the power budget levels achieved are aligned with current standards employed in the field, such as $10 \mathrm{G}$ EPON, which requires power budgets in the order of 20-24 dB for the PR10 and PR20 options. If high power budgets are required, LDPC codes could be considered, allowing to comply with the PR-30 requirements for $50 \mathrm{~Gb} / \mathrm{s}$.

\section{REFERENCES}

[1] J. L. Wei et al., "Performance and Power Dissipation Comparisons Between $28 \mathrm{~Gb} / \mathrm{s} \mathrm{NRZ,} \mathrm{PAM,} \mathrm{CAP} \mathrm{and} \mathrm{Optical} \mathrm{OFDM} \mathrm{Systems} \mathrm{for} \mathrm{Data}$ Communication Applications," IEEE J. Lightw. Technol., vol. 30, no. 20, pp. 3273-3280, 2012.

[2] M. I. Olmedo et al., "Multiband Carrierless Amplitude Phase Modulation for High Capacity Optical Data Links," IEEE J. Lightw. Technol., vol. 32, no. 4, pp. 798-804, 2014.

[3] H. Kenichi and B. Anass, "Non-orthogonal multiple access (NOMA) with successive interference cancellation for future radio access," IEICE Trans. Comm., vol. 98, no 3, p. 403-414, 2015.

[4] V. Veeravalli and A. Mantravadi, "The coding-spreading tradeoff in CDMA systems," IEEE J. Sel. Areas Comm., vol. 20, no. 2, pp. 396-408, 2002.

[5] M. Al-Imari et al., "Uplink non-orthogonal multiple access for 5G wireless networks," in Proc. of Inter. Symp. Wireless Comm. Syst., Barcelona, 2014, pp. 781-785.

[6] L. Dai, et al., "Non-orthogonal multiple access for 5G: solutions, challenges, opportunities, and future research trends," in IEEE Comm. Mag., vol. 53, no. 9, pp. 74-81, 2015.

[7] J. A. Altabas et al., "Nonorthogonal multiple access and carrierless amplitude phase modulation for flexible multiuser provisioning 5G mobile Networks," IEEE J. Lightw. Technol., vol. 35, 2017.

[8] S. Sarmiento, et al., "Experimental Investigation of 50-90 Gb/s IM DD NOMA-CAP Modulation for Short Range Optical Transmission Applications," in Proc. OECC/PSC, Fukuoka, Japan, 2019.

[9] J. Zhang et al., "Symmetrical 50-Gb/s/ $\mathrm{A}$ PAM-4 TDM-PON in O-band with DSP and Semiconductor Optical Amplifier Supporting PR-30 Link Loss Budget," in Proc. OFC, San Diego, CA, 2018.

[10] R. Bonk, "SOA for future PONs," in Proc. OFC, San Diego, CA, 2018.

[11] M. Huang et al, "Cost-effective 25G APD TO-Can/ROSA for $100 \mathrm{G}$ applications," in Proc. OFC, Los Angeles, CA, 2017.

[12] S. Sarmiento et al., "Optical Power Budget Enhancement in $50 \mathrm{~Gb} / \mathrm{s} \mathrm{IM-}$ DD PONs with NOMA-CAP Modulation and SOA-based Amplification," in Proc. ICTON, Angers, 2019.

[13] Q. Hu el al, "Up to 94 GBd THP PAM-4 Transmission with $33 \mathrm{GHz}$ Bandwidth Limitation," in Proc. ECOC, Rome, 2018. 